\title{
On How Agile Systems Gracefully Migrate Across Next-Generation Life Cycle Boundaries
}

\author{
Rick Dove \\ Stevens Institute of Technology \\ Hoboken, NJ, USA \\ 575-586-1536 \\ rick.dove@stevens.edu
}

\author{
Garry Turkington \\ Paradigm Shift International \\ Columbia, MD, USA \\ 410-868-9315 \\ garry.turkington@gmail.com
}

\begin{abstract}
In this paper we explore the nature of migration as it applies to crossing systemretirement life cycle boundaries gracefully, intending to inform purposeful design of would-be, longlived, always-effective enterprises, and the systems that support them. We use previous work on agile systems and enterprises to define migration as the crossing of a change in basic infrastructure, be it technical, organizational or strategic. We consider the classic view of the system life cycle and highlight its limitations in a rapidly changing world. We analyze two successful and contemporary large-scale migrations, and illuminate the factors that facilitated their success, contributing toward a pattern language for agile-system architecture in the process. Finally, the lessons of the analysis are suggested as potential informants for Force Transformation in the U.S. military and adoption of Service Oriented Architectures in the enterprise.
\end{abstract}

Keywords: life cycle, migration, agile enterprise, agile-system architecture, pattern language, force transformation, service oriented architecture

Rick Dove was co-Principle Investigator on the 1991 OSD/Navy-funded, industry-led study that identified and defined enterprise agility as a critical pending need. As Director of Strategy for the subsequent DARPA/NSF funded Agility Forum he organized and led the development and execution of an industry-collaborative research agenda to identify the nature of agile systems and enterprises. He is author of two books dealing with agile systems and decision making. Currently he is co-director of the Agile Systems and Enterprise graduate certificate at Stevens Institute of Technology with course development and teaching responsibilities, and pursues research in the area of self-organizing systems-of-systems. He has 25 years of start-up, turn-around, and interim executive management, including responsibilities for most C-level positions, R\&D, and major enterprise IT projects. He holds a BSEE from Carnegie Mellon University and did graduate studies at UC Berkeley in computer science.

Garry Turkington earned his BS and Ph.D degrees in computer science from the Queens University of Belfast, Northern Ireland. Since 1999 he has worked for the United Kingdom civil service in postings in both the UK and the USA. His work has focused on the design and implementation of large-scale distributed systems and the establishment of system engineering processes. His research interests are in systems and organizational agility, systems of systems, networks and distributed computing. 


\section{Introduction}

Something different is happening. Military enterprise, reacting to next generation warfare, sees the need for a new infrastructure strategy. Net centricity and force transformation encompass that plan (Cebrowski 2003, Research 2004). Business enterprise, reacting to next generation competition, sees the need for a new infrastructure. Service oriented architecture and web services encompass that plan (OASIS, W3C).

Both next-generation approaches exhibit a significant distinction from the succession of lastgenerations preceding them: they include the promise of infrastructures that will migrate through continuous change hereafter, putting an end to the traditional scrap-and-replace next-generation progression strategy.

War and competition are situated in a world of continuously increasing complexity. Complexity is a word with many interpretations - here it is used to mean more considerations for a system to deal with, e.g., technologies, interconnections, requirements, situational surprises, opportunities, etc.

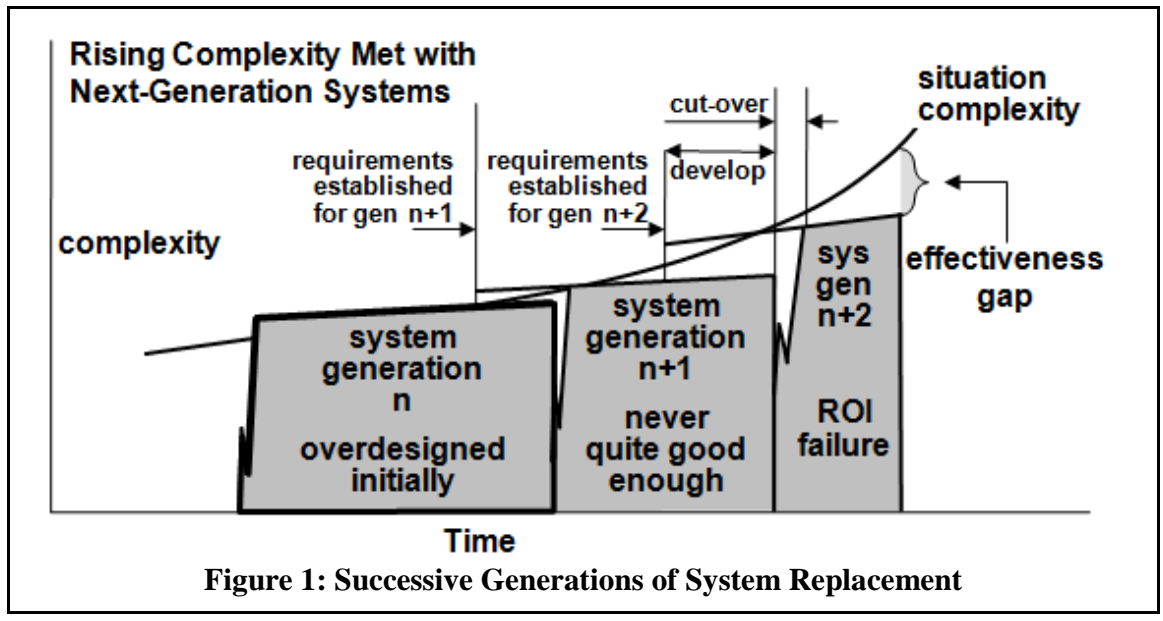
What business and military enterprise have recognized is that situational change is an accelerating continuous function, and discreet next-generation realignments, as shown in Figure 1, are now leaving gaps of unacceptable inadequacy.

The value propositions for both Force Transformation and Service Oriented Architecture is the realization of an agile enterprise that co-evolves with an unpredictable continuously changing future:

- Arthur K. Cebrowski, Director, Force Transformation (Cebrowski 2004): “...we are on course to transform our military into an agile, network-centric, knowledge-based force capable of conducting effective joint and combined military operations against all potential future adversaries. ${ }^{1}$... Transformation is foremost a continuing process. It does not have an end point. Transformation is meant to create or anticipate the future. Transformation is meant to deal with the co-evolution of concepts, processes, organizations and technology. ${ }^{2}$

- NASCIO Research Brief (NASCIO 2006): "Service Oriented Architecture: An Enabler of the Agile Enterprise ... Imagine the ability to pick and choose business and technology services. To be able to trade out services based on organizational re-design, new strategic intent, legislative requirements, or business process modifications. It is a whole philosophy about sharing, decoupling business processes from technology, to enable a fluid enterprise that can change and change quickly.

\footnotetext{
${ }^{1}$ Cebrowski 2004, opening page of document: Message from the Director, Office of Force Transformation.

${ }^{2}$ ibid, pg 8.
} 
Systems that can migrate across these next-generation gaps seamlessly have clear value. Such systems were the subject of an industry collaborative study (Nagel et al 1991, Dove 1992) in the 90s initially focused on manufacturing enterprise agility, and later broadened to encompass agility in enterprises and systems of all kinds. Interest in such systems has continued to grow: most recently with the (Upton \& Staats 2008) excellent depiction of a foreign bank's agile business-software infrastructure and (Engel \& Tyson 2008) suggesting a new way to value and justify adaptable architecture options.

Some background context on the work reported here is useful. In 2006 the increasing interest in agility as a desired enterprise and system characteristic spawned a four-course graduate certificate in Agile Systems and Enterprises at Stevens Institute of Technology, and initiated related research. The Stevens program emphasizes fundamental domain independent architecture and design principles that enable agility in systems of any kind, collectively referred to here as Response Ability Principles - or $\mathrm{RAP}^{3}$ for convenience. Discussed in the next section, RAP encompasses a set of domain independent architectural principles of structure and strategy that enable agile systems.

The work reported here is a continuation of work reported in (Dove and Turkington 2008), which cast agile system-development methods into the RAP model of domain-independent agile systems, and then looked at how the resulting architecture could inform and influence a migration from agile development into agile operations within the system life cycle. That paper concluded by indicating a number of areas for further investigation. One of the areas suggested was to continue the investigation of the RAP migration concept, with a focus on agile systems migrating across nextgeneration boundaries.

A search for suitable exemplar systems that have migrated successfully across next-generation boundaries was conducted. The principle criteria for suitableness was broad and general familiarity, so that distracting system explanations could be avoided and migration issues and concepts could be the focus. It was also decided that more than one system should be examined, and that they should not be in the same domain, in order to help obtain domain independent abstractions. Three systems ended up on the short-list: audio/video home entertainment, the Internet, and a company known to the authors that went through major business model evolutions.

To note is that all three systems fit the general signature of plug-and-play (infrastructure) dragand-drop (components) characteristic of RAP-based agile systems. No attempt was specifically made to find successful cross-generation migration in systems that do not exhibit this characteristic signature, and no suggestion is made here that such systems do not exist.

Preliminary investigation of all three systems found the company example questionable as a useful migration example - its financial performance has deteriorated recently and it was felt that this fact would obscure the successfulness of its operational migration. Home entertainment and Internet systems faired much better, appearing to provide a sufficiently different system-domain view for effective abstraction while being common enough for broad familiarity. Virtually everybody interacts with both of these systems to some extent, though generally at a more intimate level for home entertainment than Internet for many. A long ongoing migration, home entertainment systems today may contain components that were new technologies 50 years ago, integrated comfortably with new capabilities unimagined 5 years ago. More immediately, the Internet is in early stages of migration to a new packet protocol know as IPv6 (Internet Protocol version 6), which greatly expands urgently needed additional addressing capacity (Beijnum 2007, 2008).

\footnotetext{
${ }^{3}$ Response Ability Principles encompass research initiated at Lehigh University's Agility Forum in the '90s and later detailed in Response Ability (Dove 2001).
} 
The objective is to employ these two systems as exemplars for migration-enabling purposeful system design. Arguably both of these systems were not designed initially with long term broadscale migration, of the magnitude they are delivering, as a specific requirement. This quality materialized, undoubtedly through nurturing, as a fortuitous accident. For sure, the current tools of RAP theory were not formally employed in the design of these systems. How would these systems fair when cast as domain independent RAP models?

It is one thing to view these systems through the lens of RAP, and another to ask if that view illuminates the issues of agile-system migration across next-generations usefully. This, then, is the second question to resolve.

Finally, if RAP modeling provides a clear view of migration enabling concepts, how might that view inform purposeful attempts to build migration-enabled systems, such as Force Transformation and SOA (Service Oriented Architecture).

In summary, three questions drove the work reported here:

1. Can RAP tools model and depict the migration of component stereo to today's home entertainment, and the migration of the Internet from an IPv4 to an IPv6 infrastructure?

2. Can RAP casting of these two systems inform the understanding of migration in agile systems?

3. Might this RAP viewpoint of agile systems migration inform the in-process developments of SOA and Force Transformation as agile systems?

The second section, next, describes the sources and nature of the tools employed in seeking answers to these questions. The third section describes the process and results of pursuing these questions. The fourth section discusses the results and implications. The fifth and final section presents some conclusions and suggests potential further work illuminated by this effort.

\section{Experimental Setup}

Answering the three questions was conducted as an exploratory experiment. A suitable set of RAP modeling tools was selected, the literature was consulted to synthesize an Internet migration characterization, the home entertainment characterization was a refinement of common knowledge, and trial runs were conducted before the sought-after architectural conceptual patterns emerged. This section describes the source and nature of the modeling tools.

Agility as a system behavior is defined here in the broad terms intended by the 1991 Lehigh study (Nagel et al 1991, Dove 1992, Goldman et al 1995) that put the word into play: effective response under conditions of uncertainty. Subsequent research recognized that practicing such agility requires at least three aspects: situational awareness, decisive choice making, and the ability to respond. This latter aspect appeared to be the principle stumbling block observed in enterprise systems at that time. This drove a research focus at The Agility Forum on discovering architectural structure and strategy principles that would enable highly adaptable enterprise systems. This aspect of agile systems was later dubbed Response Ability and is detailed in domain independent terms in (Dove 2001). The work here deals only with this third architectural aspect of agile systems.

RAP based architecture currently encompasses seven thought-guiding frameworks: response requirements categories (2x4 elements), response performance metrics (4 elements), functional design principles (10 elements), design quality principles (3 elements), system integrity responsibilities (4 elements), an overarching architectural philosophy (3 elements), and a conceptual pattern. All except the recent addition of design quality principles are dealt with at length in (Dove 2001) and perhaps more accessibly and briefly in (Dove 2005).

Proceedings of GLOGIFT 08, June 14-16, 2008, Stevens Institute of Technology, Hoboken, NJ.

Page 4 
The conceptual pattern of RAP architecture employed here ${ }^{4}$ is one of drag-and-drop components/modules in a plug-and-play infrastructure. The overarching architectural philosophy is reusable components reconfigurable in a scalable infrastructure. Components are encapsulated oneto-one physical and functional units. Infrastructure has two parts: a passive part that provides standards (rules) for connectivity and interaction among components, and an active part that consists of four specific integrity responsibilities for maintaining and sustaining readiness for unpredictable system response needs.

Integrity responsibility, the "active" part of the infrastructure, will play a distinguishing role in determining Class 1 or Class 2 characterizations for the systems of interest. The four integrity responsibility elements are:

1) maintaining sufficient inventory of components ready for use (e.g. in home entertainment: speakers, amplifiers, tuners, tape units, etc),

2) new component addition and upgrade as new capabilities are needed (DVD units, TIVOlike appliances, wii, game consoles, etc),

3) infrastructure evolution (surround sound, video, wireless, internet, etc), and

4) assembly of components into on-demand system configurations suitable for changing needs (movement of speakers and tuners to patio, borrowing a DVD player from the family room system for the bedroom system, etc).

Four of the seven RAP frameworks are employed in the work reported here: the 3-element architectural philosophy, the migration element of the $2 \times 4$ response requirements categories, the 4element integrity responsibilities, and the plug-and-play/drag-and-drop conceptual pattern. In addition, the class distinctions between reconfigurable and reconfiguring agile systems is employed. Thus, system characteristics will be cast in the conceptual pattern of drag-and-drop components in a plug-and-play infrastructure of passive standards and active responsibilities. Exploring application of the other frameworks appears promising, but is left to subsequent work.

The 2x 4 response requirements categories are shown in Table 1. The purpose of this framework is to stimulate and organize the identification of different types of response requirements a system must be prepared to cope with effectively. Migration is one of the four proactive response domains, and is defined in (Dove 2001) as:

"Foreseen, eventual, and fundamental change. Issues are generally associated with changes to supporting infrastructure or transitions to next generation replacements." 5

Table 1: Reactive and Proactive Response Requirements Categories

\begin{tabular}{|l|l|}
\hline \multicolumn{1}{|c|}{ Reactive Response Domains } & \multicolumn{1}{c|}{ Proactive Response Domains } \\
\hline Correction - rectify a dysfunction & Creation - create/eliminate things during operation \\
\hline Variation - accommodate variable situation parameters & Improvement - improve performance metrics over time \\
\hline Expansion - add/subtract capacity & Migration - change the infrastructure significantly \\
\hline Reconfiguration - reorganize internal relationships & Modification - add/subtract capability \\
\hline
\end{tabular}

RAP thus characterizes migration as a life cycle concept for systems that hold their own in an environment of continuous situational change. The life cycle of such a system loses much of its

\footnotetext{
${ }^{4}$ (Dove 2007) distinguishes agile systems as reconfigurable (class 1) and reconfiguring (class 2). The drag-anddrop/plug-and-play pattern is most naturally associated with class 1 (reconfigurable) agile systems, as the four integrity responsibility elements of the active infrastructure are typically peopled rather than systemic.

${ }^{5}$ Dove 2001, pg 88
} 
traditionally separate stages of life cycle, characterized ${ }^{6}$ as a sequential transition through concept, development, production, utilization, support, and retirement; and instead, paces situational change with continued augmentation and evolution throughout an extended life cycle, seamlessly crossing traditionally terminal retirement boundaries into next-generation service.

Within a single generation life cycle, we have argued previously (Dove and Turkington 2008) that the distinction between development and operational stages of a system can be one of perspective rather than substance, if both exhibit a continuum of the key agile characteristics of reconfigurable components in an evolvable infrastructure. For a system to realize the benefits of agile development and operational agility its progression through the intra-system life cycle stages must be a gradual and continuous change as the nature of the components and the agents of integrity responsibility, the active portion of infrastructure, change.

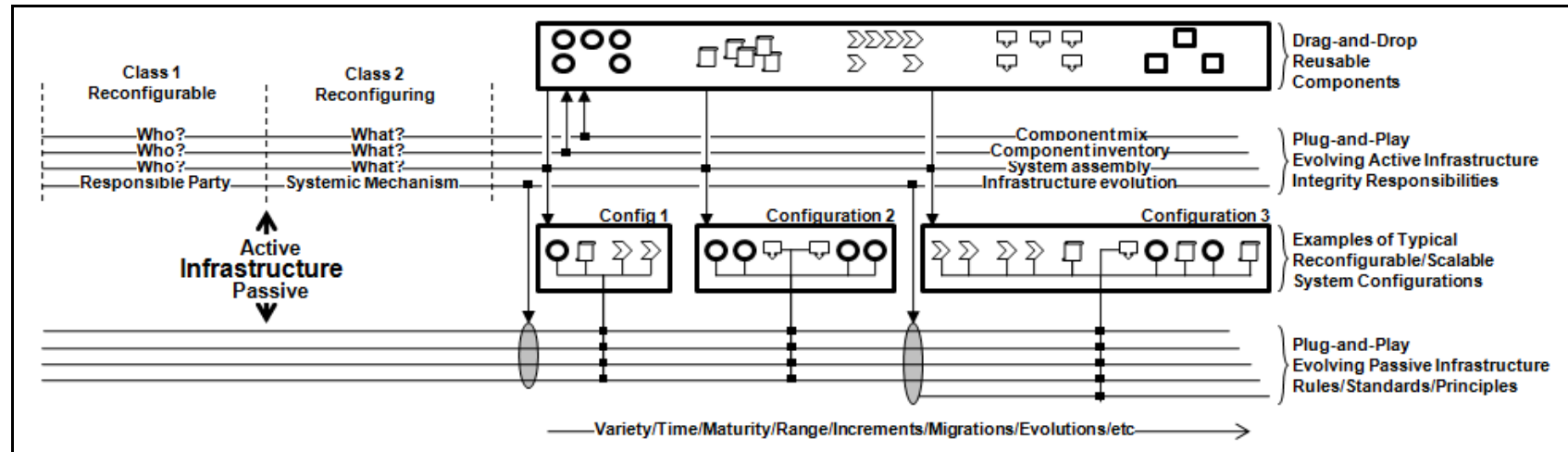

Figure 2: Generic Drag-and-Drop/Plug-and-Play Conceptual Pattern for Class 1 and Class 2 Agile Systems

Finally, the conceptual pattern depicting drag-and-drop/plug-and-play functional architecture is shown in Figure 2 for class 1 and class 2 agile systems. Note that the differences are minor, and limited to the integrity responsibilities of the active infrastructure.

More and more systems today are experiencing increasingly frequent changes in their environment - be it organizational, market, technical, strategic or otherwise. The need to respond with improved services quickly and effectively is less tolerant of a retirement and replacement strategy. Agile systems are seen as the facilitator of responsive component reconfiguration as well as infrastructure augmentation, enabling graceful migration into next-generation capabilities.

\section{Procedures and Results}

To explore this migration concept we will cast the two chosen systems into the RAP conceptual pattern. The anticipation is that this will identify and provide a clear picture of the migration enabling elements. This section will address question 1. Questions 2 and 3 will be addressed in the discussion section that follows later.

\subsection{Home Entertainment}

Since the '50s, the home-entertainment nee component-stereo industry has seen many migration events; but the underlying infrastructure concepts have remained constant throughout. Though technological advances have occurred in all types of components, the fundamental approach to physical and functional connectivity has remained conceptually constant. The core components of home entertainment systems center around the amplifier and speakers, into which multiple contentsource components connect. Though vacuum tubes have given way to transistors and vinyl records

\footnotetext{
${ }^{6}$ INCOSE 2007, pg 3.5
} 
to cassettes and on to compact discs, the role and nature of the infrastructure and the general method of connectivity have remained constant. Users could upgrade their system largely on their own schedule and according to tastes and budget. Though a new CD player would be used to best advantage with an amplifier and speakers of similar quality such was not required. This permitted a variety of system configurations ranging from the small and compact bedroom systems to the ultra high-end family room systems to evolve asynchronously and independently of technology developments and the choices made by other system owners.

As depicted in Figure 3, the leading edge migration underway currently is augmenting the infrastructure to accommodate online and multi-media capabilities. Amplifiers began servicing five

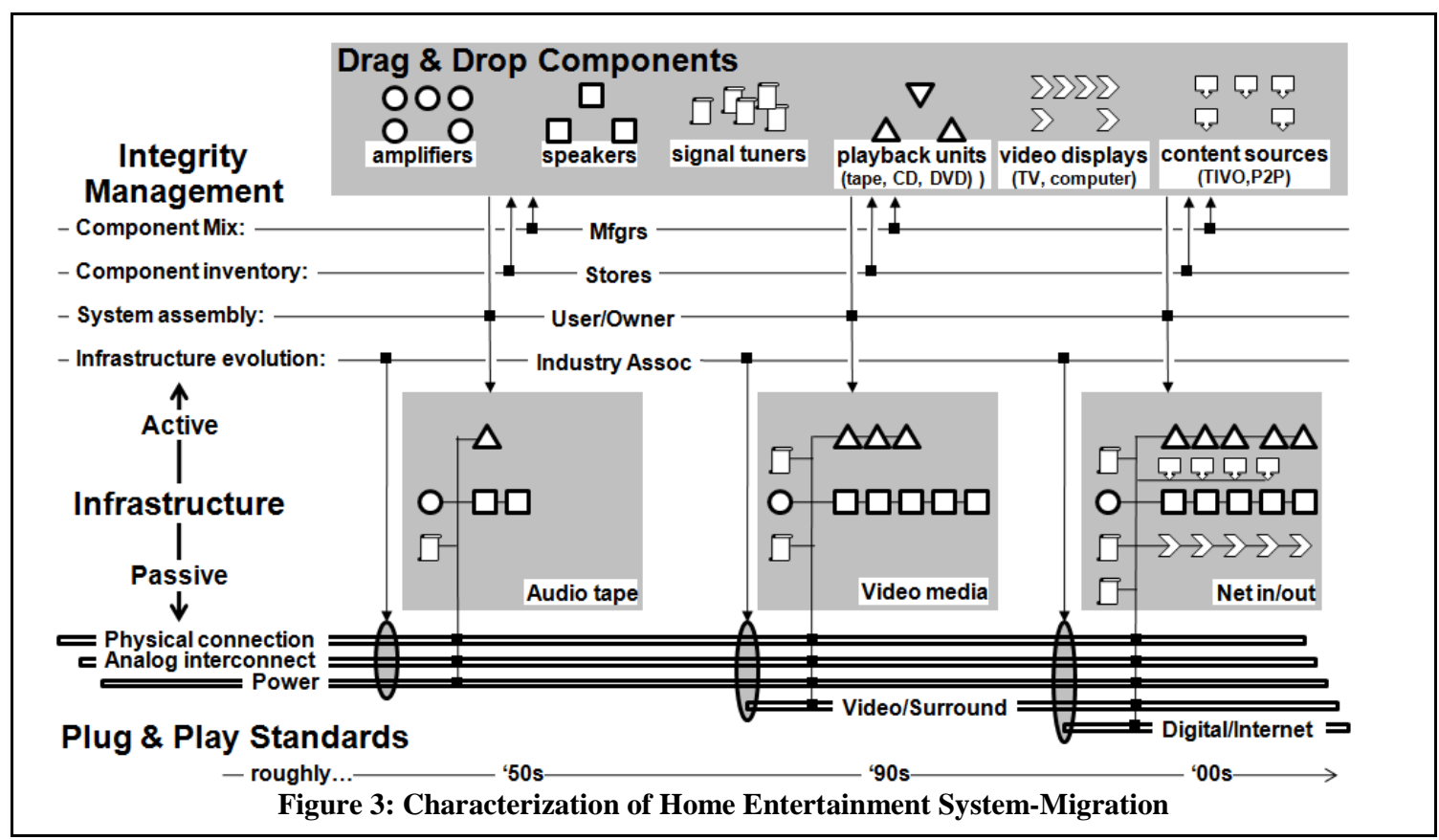

or more channels with a like number of speakers previously, about the same time as TV integration changed the system designation from HiFi stereo to home entertainment centers. That migration also brought component interconnect routing that bypassed the previous central role of the amplifier with direct connections between TV video-input and CD/DVD video sources. For historical purposes the initial generation attempts to depict the reel-to-reel audio tape deck era. Now digital video-storage and on-line Internet content are shown as the new generation of infrastructure accommodation, which also includes gaming consoles, wii devices and the current explosion of innovation. Though prior era components may be used as part of the newer systems, it is not possible to accrue the benefits of these new capabilities without augmenting the infrastructure of the previous generation.

Figure 3 depicts the casting of the Home Entertainment system in the RAP conceptual pattern image. Question 1 appears to be answered in the affirmative: Can RAP tools model and depict the migration of component stereo to today's home entertainment?

Pools of component varieties are depicted independent of life cycle or time of employment. As it was not felt germane, no attempt was made to depict the inception of new component pools or the evolution of component technologies and diversity within those pools over time, though such could easily be accommodated by showing perhaps three pools of pools, to roughly coincide with the three 
time-period configurations. Though no attempt is made to be comprehensive with categories of components, the chosen depictions are representative samples to facilitate the discussion later.

Of most interest to the migration concept, however, is the depiction of both active and passive infrastructure. RAP defines the migration response category as a significant change to infrastructure. The depiction identifies certain augmentations to the passive infrastructure as coincident with the transition across generations. This depiction is of course conceptual, and does not pretend to be precise and accurate. Its purpose is to illuminate the fundamental enabling and definitional concepts - contributing toward a pattern language (Alexander et al 1977, Cloutier 2006) for agile system architecture.

The active portion of infrastructure identifies the parties responsible for system integrity notably the actions and sustainment of reconfigurable agility. Available component mix is the responsibility of independent manufacturers, adding new types of components and better technological implementations to the mix. Component inventory is the responsibility of distribution and service channels, such as retail stores, on-line catalog sales, and repair depots; with responsibility for providing and maintaining ready-to-employ components. System configuration responsibility lies principally with the operational system owner: adding, replacing, upgrading and reconfiguring components in personal systems as and when desired. Finally, the interoperability standards and evolution of those standards that enable and facilitate plug-and-play are the responsibilities of various standards bodies supported by the industry. In general it is the intent to specifically designate the key responsible parties in the active infrastructure.

The passive portion of infrastructure is conceptually depicted, with no attempt to be comprehensive here. This part of the infrastructure is where augmentation occurs as next-generation capabilities require additional standards.

This extended tour of the Home Entertainment depiction in Figure 3 will alleviate the need to do the same depth of explanation when we look at Internet Protocol migration next.

\subsection{Internet Protocol}

The Internet Protocol (IP) is the basis of virtually all Internet traffic. Though most users are unaware of its existence, it enables web browsers to access web sites, online videos to be obtained, and email and text messaging to occur between correspondents. The current version of this protocol has been in place since 1983 when TCP/IP (Transmission Control Protocol / Internet Protocol) replaced the prior NCP (Network Control Protocol) infrastructure. The Internet Protocol version currently dominant is referred to as IPv4, signifying version 4.

The explosive growth of the Internet is quickly approaching the limits of IPv4 addressing capability. The protocol allows every device on the Internet to have a unique address; but as more and more devices are added and new types of devices become Internet-capable, the pool of available addresses is dwindling rapidly. In addition to the need for expanded addressing, security has become an issue. The Internet was created in a closed community of academics initially, when security was not critical, and consequently the security aspects of IPv4 are now seen as inadequate.

The next IP version, in early stages of deployment now, is referred to as Internet Protocol version 6, or IPv6. IPv6 provides a much greater pool of addresses, presumably better security, and other functional advantages as well. The migration to IPv6, depicted in Figure 4, is a massive undertaking, and must be achieved without the slightest interruption to existing services or obsolescence of the vast installed device population. 


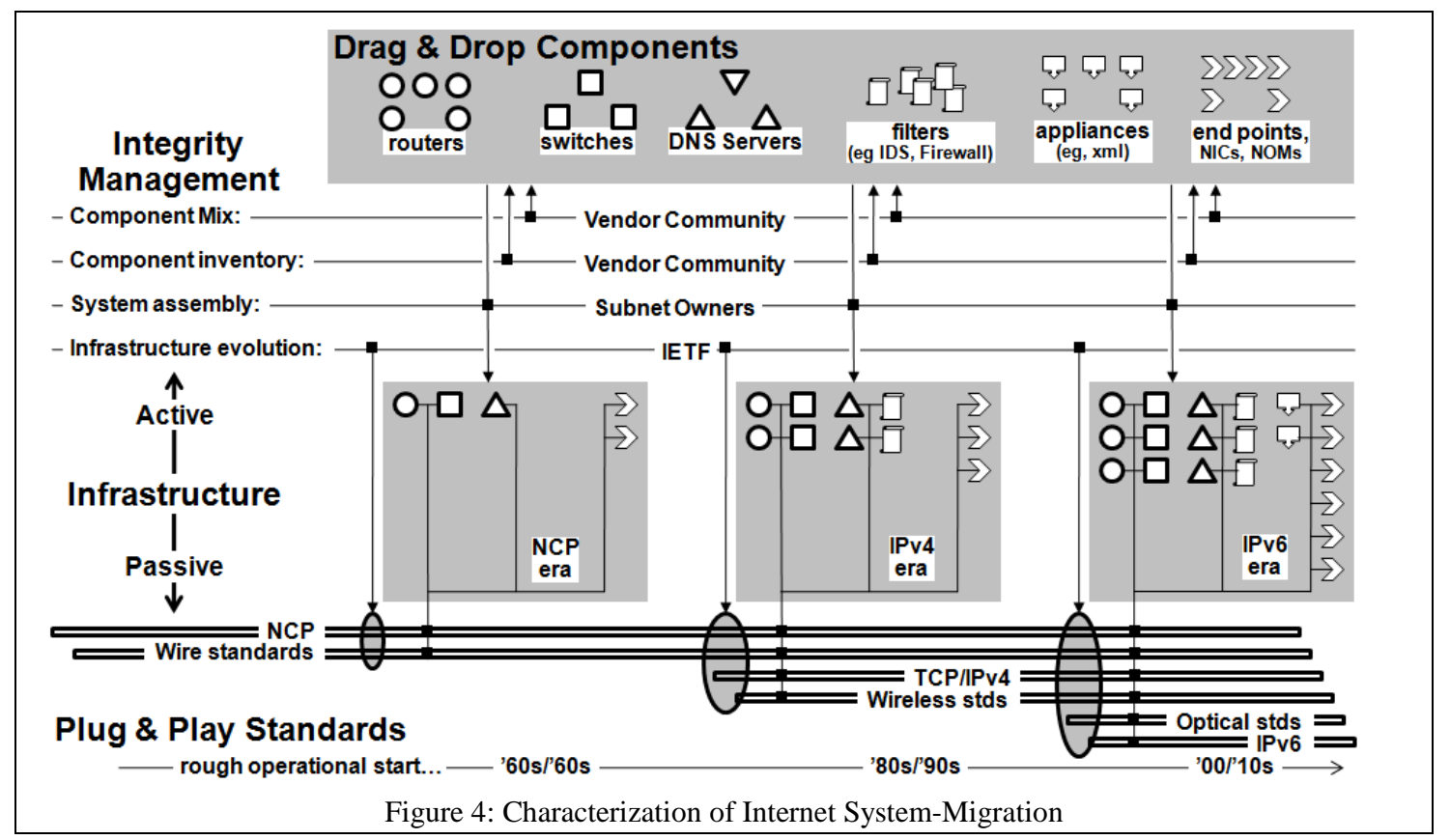

Figure 4 depicts the casting of the Internet Protocol system in the RAP conceptual pattern image. Again, question 1 appears to be answered in the affirmative: Can RAP tools model and depict the migration of the Internet from an IPv4 to an IPv6 infrastructure?

For convenience and graphic depiction brevity we appeal to poetic license and show the migration to TCP/IPv4 approximately at the same time as the migration to wireless standards. Similarly we show the augmentations of optical standards and IPv6 in time proximity. The depictions of three configuration eras makes some attempt to suggest relative sizes of component population, without concern for relative accuracies.

The passive infrastructure is represented simply - with the focus of interest on the NCP to IPv4 to IPv6 migrations; but with the recognition that other elements of infrastructure are migrating as well. The 80s/90s time frame indication on the center configuration is an attempt to peg roughly the migration to IPv4 in the ' 80 s and to wireless in the ' 90 s. Likewise the other two configurations match paired dates with paired protocol and media migrations.

The interest here again lies in the designation of responsibilities for the active portion of the infrastructure. Unlike the Home Entertainment designations, where local individuals were designated, here we designate communities for three of the four responsibilities. The difference is recognition that there is more interaction and reaction between the decisions of individual vendors and sub-net owners - where decisions by one router vendor, for instance, is likely to precipitate reactive decisions by other router vendors, somewhat like a rebalancing when portions of an ecology undergo a dominance change. As some vendors begin to favor IPv6 product creation and distribution, others are effected competitively and adjust in some fashion. Such is the case among sub-net owners as well, as more switch to IPv6 capabilities others are pressured to respond similarly in a form of self-reorganization or face the potential of becoming a backwater. This is not the case in the individual decisions of home entertainment system owners to nearly the same extent. In contrast, the evolution of infrastructure standards for internet protocol is embodied in a single central controlling organization. 


\section{Discussion and Implications}

In this section we address the two remaining questions: Can RAP casting of these two systems inform the understanding of migration in agile systems; and, Might this RAP viewpoint of agile systems migration inform the in-process developments of SOA and Force Transformation as agile systems?

\subsection{Informing the Understanding of Agile-System Migration}

Components are encapsulated capabilities-based black boxes. In Home Entertainment, for instance, components can be replaced by different brands or different technological implementations at any time without affecting the serviceability of the rest of the system. Components are encapsulated functionally and physically 1-to-1, allowing them to be inserted and deleted from a system configuration asynchronously and independently of any other component. In some cases a given component may not be able to manifest usefulness if certain other components are not present, like a DVD player present in an entertainment system without a video display of some type; but such incomplete sub-groups do not affect the ability of the rest of the system to provide service.

This encapsulation concept is a key and necessary enabler of RAP-based agile-system migration. Also key and necessary is the concept of augmentation to the passive portion of the infrastructure, as opposed to retirement or incompatible replacement of an infrastructure standard that would render existing components incapable of interoperation.

As illuminated by the modeling exercise, old infrastructure concepts and early-vintage components are not forced into retirement, and will co-exist functionally with the new; and new infrastructure pulls the usage of new capability asynchronously with the allure of benefits, rather than pushing a forced synchronous switch. Owners are free to choose when and which new values make sense to them, rather than being coerced or forced into switching when satisfaction with the status quo, untimely change, or other priorities have precedence.

This architecture has provided the home entertainment industry with a significant level of resilience. The industry has seen numerous so-called "format wars" where several manufacturers offer competing but incompatible products that provide a new type of capability. Some of these wars have seen a clear winner (Compact Disc vs Digital Audio Tape) while others have seen the new options fail to gain market traction (Super Audio Compact Disc vs DVD-Audio). Individual evolution of independent home entertainment systems have virtually no impact on the total global community of home entertainment. A next-generational replacement approach would likely have produced many more make-or-break situations where a single failure could have major negative economic effect.

For the IPv4 to IPv6 migration similar factors are at play, in that different segments of the Internet have independent life cycles and consequently may be migrated to the new version ahead of others.

The difference between a class 1 and class 2 RAP-based agile system is centrally-controlled sustainment vs. self organizing sustainment. In the one case specific people or committees (for instance) with centralized sustainment responsibilities can be named, in the second case sustainment is caused by the equilibrium-seeking self-reorganization of decentralized interactions among autonomous agents. It is thought that the Home Entertainment system fits more the model of class 1, as the owner that configures systems very centrally controls the system configuration, and has little effect or influence on owners of other Home Entertainment systems. Migration of the Internet Protocol, in contrast, has a greater degree of coupling between the migration-deciding agents. As subnets opt for IPv6 profiles, other interconnected subnets may become shunned for services of lesser security or less optimal interaction. 


\subsection{Potential for Informing SOA and Force Transformation Adoptions}

It is observed that SOA and Home Entertainment environments share a characteristic that may be useful in guiding SOA adoption plans. Both occur in relative isolation to their greater communities, and resemble a class 1 agile system. Force Transformation, on the other hand, has an environmental profile more like the Internet Protocol model. Both have sizable sub-groups with interdependent couplings - looking somewhat like an ecological system in the large.

Adoption and subsequent migratory evolution of SOA within an enterprise is largely a local decision, with little interdependence on when and what other enterprises choose to do. Though enterprises are increasingly networked to each other electronically as well as strategically, SOA is largely an internal infrastructure for enterprise IT support of business practices. Perimeter gateways of various types are standard methods for reconciling inter company transactions. The nature of the SOA infrastructure nevertheless must conform to greater community common/universal standards if maximum and sustainable access to component services of benefit are to be realized. This raises a cautionary flag on brand-unique infrastructure employment, as well as enterprise- or brand-unique service interfaces.

Force Transformation is a massive undertaking, on many functional fronts within each military force as well as across the many independent but interdependent military forces of Army, Navy, Air Force, Marines, and Coast Guard. Force Transformation is predicated on developing far more intimate interoperability than currently exists. The magnitude of the effort necessarily requires an asynchronous adoption for economic, cultural and technological reasons as a minimum - without any disruption of capability. The military has a tradition of controlled mandated actions that may not serve well in either the initial adoption or the subsequent continual evolution intended. The model of Internet Protocol migration that relies on pulling self-organized adoption with enticing benefit, rather than forcing a change that may be incompatible with the reality of the status quo, might well provide both economic and speed-of-adoption advantages.

These small observations and suggestions are as far as the current work warrants, but they have provided an interesting foundation for further investigation in follow-on work.

\section{Concluding Remarks}

This work presented a generic conceptual pattern for RAP-based agile systems capable of depicting key agility and migration enabling elements, independent of the system domain. The nature of, and differences between, class 1 (reconfigurable) and class 2 (reconfiguring) RAP-based agile systems was introduced. It was suggested that the RAP conceptual pattern tool contributes toward the development of a pattern language for agile systems architecture. Migration in a RAPbased agile system was defined as a change to infrastructure facilitating a graceful crossing of nextgeneration capability boundaries. Two RAP-depicted systems that are successfully providing graceful migration were explored, looking for suggestions of domain-independent fundamental enabling concepts. Finally, it was suggested that the fundamental concepts that appear to enable and facilitate this migration capability might usefully inform purposeful attempts - at designing agile systems for graceful migration across next-generation boundaries.

No claim is made that these observations and suggestions form a sufficient or complete theory of migration in RAP-based agile systems. This work is expected to stimulate further research and application, based on its demonstrated ability to offer a pattern language for exploration and discussion of systems' agility and, specifically, graceful migration across next-generation boundaries. 
System life cycle concepts have served us well in system engineering understandings and activities. Clearly there is a time before a system exists, a time when it is created, a time when it is in service, and a time when it no longer exists in any functional capacity. The concepts of investment and return on investment (ROI) are an integral part of life cycle, though usually blurred across the activity boundaries that characterize life cycle stages.

The pace of technology has continued to accelerate, as has the interconnection and interaction of human society and its support systems. All have reached a speed of change that conspire against an ROI for systems not made to freely evolve. The result is a process of natural selection: systems built for evolution will become the dominant concept. Some are already here, more by accident than purposeful design. The need for systems with longer life has reached the conscious system engineering level, and the search for understanding is underway.

Life cycle models will necessarily need updating to recognize continuous revitalization activity. Systems will retain identity across major functional transformations that would have spelled retirement and replacement. ROI will likewise need new math underpinnings as well. All are subjects for research, development, and natural selection.

This work is part of a larger body of research exploring a RAP-based theory of agile systems. It is stimulated primarily by promising masters and doctoral student course-projects that warrant further collaborative development.

\section{References}

Alexander, C., Ishikawa, S., Silverstein, M., with Jacobson, M., Fiksdahl-King, I. and Angel, S. (1977), A Pattern Language: Towns, Buildings, Construction, Oxford University Press.

Beijnum, I. (2007), “Everything You Need to Know About IPv6,” ars technica, March, http://arstechnica.com/articles/paedia/IPv6.ars.

Beijnum, I. (2008), “The night the IETF turned off IPv4,” ars technica, March 2008, http://arstechnica.com/articles/culture/ietf-ipv6-switchoff.ars.

Cebrowski, A.K. (2004), Military Transformation: A Strategic Approach, U.S. Department of Defense, Office of Force Transformation.

Cloutier, R. (2006), Applicability of Patterns to Architecting Complex Systems, Ph.D. Dissertation, Stevens Institute of Technology, Hoboken, N.J., USA.

Dove, R. (1992), "What's All This Talk About Agility - The $21^{\text {st }}$ Century Manufacturing Enterprise Strategy", Prevision, Japan Management Association, www.parshift.com/Files/PsiDocs/Rkd92Art6.pdf.

Dove, R. (2001), Response Ability - The Language, Structure, and Culture of the Agile Enterprise, Wiley.

Dove, R. (2005), "Fundamental Principles for Agile Systems Engineering,” Conference on Systems Engineering Research (CSER), Stevens Institute of Technology, Hoboken, NJ, www.parshift.com/Files/PsiDocs/Rkd050324CserPaper.pdf.

Dove, R. (2007), SDOE 678 and 683 course materials, Stevens Institute of Technology, School of Systems and Enterprises, available by request to rick.dove@stevens.edu.

Dove, R. and Turkington, G. (2008), "Relating Agile Development to Agile Operations," Conference on Systems Engineering Research (CSER), University of Southern California, Redondo Beach, April, www.parshift.com/Files/PsiDocs/Pap080404Cser2008DevOpsMigration.pdf.

Engel, A. and Tyson, B. (2008), "Designing Systems for Adaptability by Means of Architecture Options,” forthcoming in Systems Engineering, 11(2), www.incose.org/symp2008/dmdocuments/paper_example01.pdf.

Goldman, S., Nagel, R., Preiss, K. (1995), Agile Competitors and Virtual Organizations, Van Nostrand Reinhold, 1995.

INCOSE (2007), Systems Engineering Handbook - A Guide For System Life Cycle Processes and Activities, Version 3.1, International Council on Systems Engineering, August,

Nagel, R., Dove, R., Goldman, S., Preiss, K.. (1991), 21st Century Manufacturing Enterprise Strategy, Volumes 1 and 2, Coolingdale, PA: DIANE Publishing Company, USA.

NASCIO (2006), Service Oriented Architecture: An Enabler of the Agile Enterprise, National Association of State Chief Information Officers.

OASIS (n.d.), Committee Categories: SOA, Organization for the Advancement of Structured Information Standards, www.oasis-open.org/committees/tc_cat.php?cat=soa. 
Research, Evidence Based, (2003), Network Centric Operations Conceptual Framework, Prepared for John Garstka Office of Force Transformation by Evidence Based Research, Inc., Vienna VA, November, www.oft.osd.mil/library/library files/document_353_NCO\%20CF\%20Version\%201.0\%20(FINAL).doc.

Upton, D. and Staats, B. (2008), "Radically Simple IT," Harvard Business Review, March. W3C, Web Services Activities, World Wide Web Consortium.

W3C (2004) Web Services Architecture, World Wide Web Consortium. www.w3.org/TR/wsarch/. 